\title{
1 Influence of grade of obesity on the achievement of VO2max using an 2 incremental treadmill test in youths
}

3 Louis Toulouse ${ }^{\mathrm{a}}$, Patrick Mucci ${ }^{\mathrm{b}}$, Thierry Pezé ${ }^{\mathrm{a}}$, Gautier Zunquin ${ }^{\mathrm{c}}$.

5 a Unité de Recherche Pluridisciplinaire Sport Santé Société, ULR 7369 - URePSSS -

6 Univ. Littoral Côte d'Opale, Univ. Lille, Univ. Artois, F-59000 Lille, France

7 b Unité de Recherche Pluridisciplinaire Sport Santé Société,Univ. Lille, ULR 7369 -

8 URePSSS - Univ. Littoral Côte d'Opale, Univ. Artois, F-59000 Lille, France

9 c Laboratoire Mouvement, Equilibre, Performance, Santé. Campus Montaury, EA 4445

10 - Université Pau Pays de l'Adour, F-64600 Anglet, France

11

$12{ }^{*}$ Corresponding author: Louis Toulouse, Unité de Recherche Pluridisciplinaire Sport Santé

13 Société, EA 7369 - URePSSS - Univ. Littoral Côte d'Opale, Univ. Lille, Univ. Artois, F-59000 Lille,

14 France. E-mail: louis.toulouse@etu.univ-littoral.fr

15 ORCID : 0000-0003-1215-7104 
Achievement of $\mathrm{VO}_{2 \max }$ in youths with obesity

17 Influence of grade of obesity on the achievement of VO2max using an 18 incremental treadmill test in youths

The purpose of this study was to analyze the influence of grade of obesity on the probability of achieving a $\mathrm{VO}_{2}$ plateau and threshold secondary criteria for verifying $\mathrm{VO}_{2 \max }$ during a treadmill walk test in youths with obesity. Therefore, 72 youths with obesity (aged 8-16) performed an incremental treadmill walk test to exhaustion during which oxygen uptake $\left(\mathrm{VO}_{2}\right)$, minute ventilation (VE), heart rate (HR) and rating of perceived exertion were continuously measured. HR corresponding to a "hard" level of perceived exertion was reported and expressed as a percentage of the predicted $\mathrm{HR}_{\max }$. The rate of achievement of criteria for validation $\mathrm{VO}_{2 \max }\left(\mathrm{VO}_{2}\right.$ plateau; $\mathrm{HR}>95 \%$ theoretical $\mathrm{HR}_{\max }$; $\mathrm{RER}>1.0$; rating of perceived exertion $\geq$ "hard") was compared between participants with grade I and grade II obesity. $37 \%$ of the participants achieved a $\mathrm{VO}_{2}$ plateau and $23 \%$ achieved both an HR>95\% and RER $>1.0$. Youths with grade II obesity had lower minute ventilation $(\mathrm{p}<0.01)$ tended to be more likely to reach an $\mathrm{HR}>95 \%$ $(\mathrm{OR}=0.33 ; \mathrm{P}=0.06)$ and a "hard" rating of perceived exertion than grade I (OR $=4.5 ; \mathrm{P}=0.07)$. However, there was no influence of grade of obesity on the achievement of $\mathrm{VO}_{2}$ plateau, and RER>1.0. A higher grade of obesity was related to a lower peak in minute ventilation normalized by fat-free mass, lower peak heart rates and premature reaching of a "hard" rating of perceived exertion. Therefore, youths with higher grades of obesity might be less likely to display maximal heart rate during an incremental treadmill test, probably due to higher ventilatory constraints and higher ratings of perceived exertion.

Keywords: children ; adolescents ; obesity ; treadmill ; $\mathrm{VO}_{2 \max }$

Words count (text only) : 3102

Number of table : 2 
Achievement of $\mathrm{VO}_{2 \max }$ in youths with obesity

\section{INTRODUCTION}

45 Cardiorespiratory fitness is one of the best predictors of all-cause mortality in adults ${ }^{1}$, 46 especially when it is normalized by fat-free mass ${ }^{2}$. Cardiorespiratory fitness is assessed

47 by the maximal oxygen uptake $\left(\mathrm{VO}_{2 \max }\right)$, which is the highest value of $\mathrm{VO}_{2}$ clinically attainable during a progressive cycling or treadmill test conducted to exhaustion ${ }^{3}$. Since walking is more representative of free-living physical activities, treadmill walk tests are mostly used to assess the $\mathrm{VO}_{2 \max }$ in youth with obesity ${ }^{4-6}$. During these tests, the speed is constant and usually between 3.5 and $5 \mathrm{~km} \cdot \mathrm{h}^{-1}$, which is close to the preferred walking speed in this population ${ }^{7}$. The intensity is increased by increasing the slope, amounting to 1 or $2 \%$ per minute ${ }^{4,5}$. To verify the achievement of $\mathrm{VO}_{2 \max }$ (i.e., a maximal aerobic effort) during this type of test, it was proposed that youths with obesity should reach at least one of the three following physiological criteria: 1) the presence of a $\mathrm{VO}_{2}$ plateau; 2) a heart rate $>200 \mathrm{bpm}$ or $95 \% \mathrm{HR}_{\max }$; 3) a respiratory exchange ratio > 1.0. According to these criteria, Gutin et al. found that 66 out of 80 participants $(\sim 83 \%)$ reached $\mathrm{VO}_{2 \max }{ }^{4}$. Using the same protocol, a study ${ }^{6}$ found that $29 \%$ of adolescents with grade II obesity achieved a $\mathrm{VO}_{2}$ plateau. However, the rates of achievement of

60 secondary threshold criteria were not specified. Finally, one study ${ }^{5}$ did not specify the 61 criteria $^{4}$ used to verify the achievement of $\mathrm{VO}_{2 \max }$, which does not allow to determine the extent to which their treadmill protocol elicited a maximal aerobic effort. Since

$63 \mathrm{VO}_{2 \max }$ remains the gold standard for monitoring aerobic fitness in youths with obesity ${ }^{8}$, 64 it is necessary to ensure that a maximal aerobic effort has been made during the test. 65 However, there is still a lack of transparency regarding the criteria used to ensure that a 66 maximal aerobic effort has been made during treadmill walk tests in youths with 67 obesity. Furthermore, no study has investigated the influence of grade of obesity on the probability of achieving $\mathrm{VO}_{2 \max }$ during this type of protocol. Thus, further studies are 
needed to better understand the mechanisms associated with the non-achievement of a

$70 \mathrm{VO}_{2}$ plateau and secondary threshold criteria for verifying $\mathrm{VO}_{2 \max }$ in youth with 71 obesity.

72 Therefore, this study aimed to assess the influence of grade of obesity on the probability

73 of achieving a $\mathrm{VO}_{2}$ plateau and secondary threshold criteria for verifying $\mathrm{VO}_{2 \max }$ during 74 an incremental treadmill walk test in youth with obesity.

\section{METHODS}

\section{Participants}

7772 youths with obesity were included in this study. The inclusion criteria were an age 78 between 8 and 16 years old and a BMI superior to the $85^{\text {th }}$ percentile of the IOTF 79 standards for BMI, defining grade I obesity in youths ${ }^{9}$. These youths are oriented by 80 their practitioner towards obesity management networks (including the hospital center 81 and associations) where they are offered to follow care adapted to their needs. During 82 their inclusion appointment to the program, the youths participants and their parents 83 received an oral and written explanation of the experimental procedure and the 84 objectives of the study. For youths, the information notice was written in a language 85 adapted to their level of understanding. After a reflection time of at least one week 86 following the inclusion visit, the youths and the voluntary parents signed a written 87 consent summarizing the information relating to the study.

\section{General procedure}

89 The experiments took place in the department of pulmonary medicine of the hospital

90 center in the presence of a specialist doctor. Participants were called in the morning 91 between 10 a.m. and 12 p.m. or in the afternoon between 3 p.m. and 7 p.m., at least two 
92 hours after their last food intake to avoid digestive discomfort during the test. Parents

93 were asked to make sure that their youths did not exercise intensively during the 48

94 hours preceding the appointment. Before the experiments, the participants were

95 measured in standing and seated positions using a stadiometer. Then, the body weight

96 and composition were measured using bioimpedance-analysis (Tanita DC-360, Japan).

97 These measurements allowed us to obtain an estimate of their body composition (fat-

98 free mass and fat mass) and to calculate their BMI z-score based on the $\mathrm{IOTF}^{9}$ and

$99 \mathrm{WHO}^{10}$ standards. Participants who had a BMI z-score between 1 and 2 (not included)

100 according to the IOTF standards were categorized into "grade I obesity", whereas those

101 who had a BMI z-score > 2 were categorized into "grade II obesity". From the

102 anthropometrical measurements, the Age of Peak Height Velocity - age of PHV (also

103 called "Maturity Offset") was estimated using the following sex-specific equations

104 provided by Mirwald et al. ${ }^{11}$ :

Eq. $1:$ Maturity Offset (boys) $=-9.236$

$+0.0002708 \cdot$ Leg Length and Sitting Height interaction

$-0.001663 \cdot$ Age and Leg Length interaction

$+0.007216 \cdot$ Age and Sitting Height interaction

$+0.02292 \cdot$ Weight by Height ratio

105

$$
\begin{gathered}
\text { Eq. } 2: \text { Maturity Offset }(\text { girls })=-9.376 \\
+0.0001882 \cdot \text { Leg Length and Sitting Height interaction } \\
+0.0022 \cdot \text { Age and Leg Length interaction } \\
+0.005841 \cdot \text { Age and Sitting Height interaction } \\
-0.002658 \cdot \text { Age and Weight interaction } \\
+0.07693 \cdot \text { Weight by Height ratio }
\end{gathered}
$$


Achievement of $\mathrm{VO}_{2 \max }$ in youths with obesity

107 Then, age from the peak height velocity (age from PHV) was estimated by subtracting

108 the estimated age of PHV by the chronological age. A negative value indicates that the

109 PHV has not occurred yet ${ }^{11}$. The maturiy offset and age from PHV were used as an

110 indicator of the maturity of the participants.

\section{Incremental exercise}

112 The incremental treadmill protocol consisted of performing a 3-minute warm-up at $0 \%$

113 slope and a speed between 3.0 to $4.5 \mathrm{~km} \cdot \mathrm{h}^{-1}$ so that the participant reached a heart-rate

114 (HR) steady-state at $60 \pm 5 \%(110-130 \mathrm{bpm})$ of the predicted maximum HR estimated

115 with the formula of Tanaka ${ }^{12}[\mathrm{HRmax}=208-0.7 *$ age $]$. Normalizing the starting

116 speed by the HR was intended to ensure an equitable starting intensity between

117 individuals and optimal exercise duration (> 5 minutes excluding warm-up) for all

118 participants ${ }^{13}$. During the first 5 minutes, the slope increased by $2 \%$ per minute and the

119 speed was kept constant. From the sixth minute, the slope was maintained at $10 \%$ and

120 the speed increased by $0.3 \mathrm{~km} \cdot \mathrm{h}^{-1}$ for a starting speed between $3.0 \mathrm{~km} \cdot \mathrm{h}^{-1}$ and $3.7 \mathrm{~km} \cdot \mathrm{h}^{-1}$

121 or by $0.4 \mathrm{~km} \cdot \mathrm{h}^{-1}$ for a starting speed between 3.7 and $4.5 \mathrm{~km} \cdot \mathrm{h}^{-1}$. This protocol was

122 selected because high treadmill grades would be associated with an increased failure to

123 achieve $\mathrm{VO}_{2 \max }{ }^{14}$. The test ended when the participant declared that he was no longer

124 able to continue the test despite the encouragement of the experimenter.

\section{Measurement and processing of data}

126 During exercise, HR was measured using a recently validated optical heart rate monitor

127 (Polar $\mathrm{OH} 1$, Finland) ${ }^{15}$ positioned at the left wrist. The peak heart rate $\left(\mathrm{HR}_{\text {peak }}\right)$ 128 observed during the test was the highest value averaged on 3 points. The $\mathrm{VO}_{2}$ was 129 measured breath-by-breath using a portable respiratory gas analyzer $\left(\right.$ Cosmed $\mathrm{K} 4 \mathrm{~b}^{2}$, 
130 Italy) calibrated before the exercises following the manufacturer's recommendations.

131 The $\mathrm{VO}_{2 \text { peak }}$ and peak in minute ventilation $\left(\mathrm{VE}_{\text {peakBM }}\right)$ were reported as the highest

132 values over a 30 s moving-averaged interval, as recently recommended in youths ${ }^{16}$.

133 Rating of perceived exertion (RPE) was continuously assessed using the CR-10 Borg

134 scale previously used in youths with obesity ${ }^{5}$. This scale describes the difficulties of

135 breathing from level 1 ("not at all") to 10 ("maximal"). The HR measured when the

136 participant declared a level of $5 / 10$ on the scale - corresponding to the "hard" item

$137\left(\mathrm{HR}_{\mathrm{RPEhard}}\right)$ - was reported and expressed as a percentage of the individual predicted

$138 \mathrm{HR}_{\max }$. We chose this indicator to get information about the level of physiological stress

139 that each participant was able to tolerate before the effort was "hard", a term that has

140 more clinically significance in youths ${ }^{17}$.

\section{Criteria for verifying $\mathrm{VO}_{2 \max }$}

142 Following previous studies in youths with obesity ${ }^{4,6}$, the present cardiorespiratory-based

143 criteria for verifying $\mathrm{VO}_{2 \max }$ were used: primary criterion = presence of a $\mathrm{VO}_{2}$ plateau;

144 secondary threshold criteria $=\mathrm{HR}>95 \%$ of theoretical maximal HR and RER $>1.0$.

145 Participants were considered as $\mathrm{VO}_{2 \max }$ achievers when at least the $\mathrm{VO}_{2}$ plateau or both

146 the secondary threshold criteria were met. The presence of a $\mathrm{VO}_{2}$ plateau was

147 characterized when the increase in $\mathrm{VO}_{2}$ during the last completed minute of effort was

148 strictly less than $50 \%$ of the increase observable during the penultimate minute of effort.

149 This method based on the individual workload/ $\mathrm{VO}_{2}$ relationship was recently

150 recommanded ${ }^{18}$ and used to detect $\mathrm{VO}_{2}$ plateaus in adults with obesity ${ }^{19}$. A fourth

151 criterion based on the achievement of an RPE $\geq 5$ (item "hard") was also used. This

152 criterion was used as a measurement of the psychological tolerance to the treadmill test.

153 Conversely, the criteria based on volitional exhaustion was not used because the latter is 
154 determined rather by the experimenter than the participant, limiting the possibilities of

155 inter-study comparisons.

\section{Statistical analysis}

157 The data were analyzed on the $\mathrm{R}$ software (version 3.6.2). The normality and 158 homogeneity of the distributions were checked respectively with the Shapiro-Wilk test

159 and the F test of Snedecor. Firstly, the clinical characteristics and peak exercise data of

160 participants were compared between those who had grade I and those who had grade II

161 obesity using Student t-tests and Cohen's $d$. Secondly, the number and rate of

162 participants who met the criteria for verifying $\mathrm{VO}_{2 \max }$ were calculated and reported 163 according to the grade of obesity. Chi-Square tests were performed to analyze the

164 differences in the rate of achievement of each criterion according to the grade of 165 obesity. If significant differences were found, logistic regression analysis was 166 performed to examine the association between the grade of obesity and the achievement 167 of each criterion. The significance threshold was set at $P<0.05$.

\section{Results}

170 Participants' characteristics, cardiorespiratory, and perceptual responses are shown in

171 table 1. Youths with grade II obesity were less mature $(P<0.05, d=0.5)$, heavier $172(d=1.25)$ and had a higher BMI z-score $(d=4.47)$ and percentage of body fat $(d=2.38)$

173 than grade $\mathrm{I}(P<0.001$ for each parameter $)$. VE $\mathrm{E}_{\text {peak }}$ divided by body mass $(P<0.001$, $174 d=1.34)$, fat-free mass $(P<0.05, d=0.72)$ and $\mathrm{VO}_{2 \text { peak }}$ divided by body mass $(P<0.01$, $175 d=1.03$ ) were lower in youths with grade II obesity than grade I. Conversely, no 
176 between-groups significant differences were found regarding $\mathrm{HR}_{\text {peak }}, \mathrm{HR}_{\mathrm{RPEhard}}$, and

$177 \mathrm{RER}_{\text {peak }}$.

178

*** Table 1 here $* * *$

179 Table 2 summarizes results regarding the achievement of both primary and secondary

180 threshold criteria for verifying $\mathrm{VO}_{2 \max }$ in grade II vs. grade I obesity. A $\mathrm{VO}_{2}$ plateau

181 was met by $38 \%$ of the participants and $23 \%$ met both an $\mathrm{HR}>95 \% \mathrm{HR}_{\max }$ and anan $\mathrm{R}$

182 RER>1.0. According to the criteria for verifying $\mathrm{VO}_{2 \max }$ used in children with obesity,

$18354 \%$ of the participants would have reached $\mathrm{VO}_{2 \max }$ during the test. An RPE>5/10 was

184 reached by $92 \%$ of the participants.According to the Chi-square tests, youths with grade

185 II obesity tended to be less likely to display an $\mathrm{HR}>95 \% \mathrm{HR}_{\max }(\mathrm{OR}=0.33 ; P=0.06)$

186 and more likely to indicate an $\mathrm{RPE}>5 / 10(\mathrm{OR}=4.5 ; P=0.07)$ than grade $\mathrm{I}$. However, no

187 significant differences were found regarding the achievement of a $\mathrm{VO}_{2}$ plateau

$188(P=0.33), \mathrm{RER}>1.0(P=0.27)$, or $\mathrm{VO}_{2 \max }(P=0.44)$.

$189 * * *$ Table 2 here $* * *$

190 Discussion

191 The main purpose of this study was to study the influence of the grade of obesity on the

192 probability to meet the primary and secondary threshold criteria for verifying $\mathrm{VO}_{2 \max }$

193 during a treadmill test. The secondary purpose was to measure the cardiorespiratory and

194 perceptual responses to this test in the youth with obesity, which is an under-evaluated

195 population. The main findings of this study are that 1) youths with grade II obesity were

196 less likely to display an $\mathrm{HR}>95 \% \mathrm{HR}_{\max }$ and 2) youths with grade II obesity were more

197 likely to indicate an RPE reflecting a "hard" level of effort than grade I. Furthermore, 
198 youth with grade II obesity displayed lower peaks in minute ventilation normalized by

199 the fat-free mass than grade I.

201 Adults suffering from chronic diseases have a lower ability to achieve $\mathrm{VO}_{2 \max }$ during a 202 progressive test conducted until exhaustion ${ }^{20}$. However, in youths with obesity, current 203 studies provide little data regarding the achievement of criteria for verifying $\mathrm{VO}_{2 \max }{ }^{4-6}$. 204 Therefore, knowledge about the individual's factors associated with the probability of 205 achieving $\mathrm{VO}_{2 \max }$ remains limited. Although no statistically significant, the present 206 results in a relatively large sample of youth with obesity $(n=72)$ suggest that the grade 207 of obesity may be associated with a lower probability of meet an $\mathrm{HR}>95 \% \mathrm{HR}_{\max }$ and a 208 higher probability of meet a "hard" level of RPE. Thus, youths with higher grades of 209 obesity might perceive the test as more difficult. This result may be of significance 210 since perceptual responses play a major role during a progressive test conducted until 211 exhaustion $^{20}$. The present data would be supporting those of our predecessors ${ }^{5}$ who 212 found that RPE was higher in grade II than grade I obesity at the end of a treadmill test.

213 They hypothesized that these differences could be due to an increased aerobic energy 214 cost, a greater $\mathrm{CO}_{2}$ turnover, and a lower anaerobic threshold in grade II obesity. In the 215 present study, the peak in minute ventilation normalized by body mass was significantly 216 lower in youths with grade II than grade I obesity, and this differences persisted even 217 after removing differences in body composition. This could mean that ventilatory 218 adaptations to exercise were lower in grade II vs. grade I. Interestingly, it has been 219 previsouly suggested that lower cardiorespiratory fitness in youths with obesity could be 220 partly explained by ventilatory factors ${ }^{21}$. The excess adipose tissue at the thoracic level 221 would constrain them to breathe at low lung volumes, increasing the work of respiratory 
222 muscles to the detriment of locomotor muscles ${ }^{21}$. Nevertheless, ventilatory factors

223 would have a little impact on cardiorespiratory fitness in youths ${ }^{21}$. Accordingly, no

224 differences were found between grade I and grade II regarding $\mathrm{VO}_{2 \text { peak }}$ after removing

225 differences in body mass and composition. Therefore, the present data would be in

226 accordance with the previous hypothesis ${ }^{5}$ that ventilatory factors are involved in the

227 higher feeling of difficulty of youths with grade II obesity, but has limited influence on

$228 \mathrm{VO}_{2 \text { peak }}^{21}$. Otherwise, breathing difficulties could also explain that youths with grade II

229 obesity were less likely to met an $\mathrm{HR}>95 \% \mathrm{HR}_{\max }$. It should be noted however that the

230 relative $\mathrm{HR}$ at which the RPE was 5/10 ("hard") was higher than what could be

231 expected, supporting previous data showing that RPE is underestimated in youths with

232 obesity $^{5,22,23}$

233 Other factors may explain that youths with higher grades of obesity perceive their effort

234 as more difficult. Firstly, obesity has many deleterious consequences on the

235 musculoskeletal function of the lower limbs in youths ${ }^{24}$. Thus, lower limb pain

236 sensations may contribute to the higher RPE in those who have obesity. Considering the

237 low RPE scores found at the end of an incremental running or walking fitness test ${ }^{5,21,22}$,

238 it could be relevant to use an RPE scale based on muscular sensations. Indeed, current

239 RPE scales are based on cardiorespiratory sensations ${ }^{25}$, assuming that cardiorespiratory

240 function is the limiting factor during an aerobic fitness test. Conversely, the

241 combination of both a "local" and "overall" RPE scale would make it possible to

242 dissociate the chest sensations from the lower limb sensations, which could help

243 clinicians to better identify the reason for stopping the test. This approach has already

244 been used in healthy children using an incremental cycling test and showed that "leg

245 RPE" was significantly higher than "overall RPE" and "chest RPE". ${ }^{26}$ Secondly, the

246 lack of experience in vigorous-intensity exercises (due to poor engagement in physical 
247 activities) is known to negatively affect RPE measurements ${ }^{27}$. However, physical

248 activity levels were not measured in this study, limiting further interpretation of current 249 data.

250 The probability of achieving $\mathrm{VO}_{2 \max }$ using a treadmill protocol according to the grade of

251 obesity has previously been studied in women ${ }^{19}$. In accordance with the present results 252 in youths, the authors found no differences between grade I and grade II obesity women 253 regarding the achievement of a $\mathrm{VO}_{2}$ plateau. However, they also reported no differences 254 regarding the achievement of secondary threshold criteria (i.e., $\mathrm{HR}>95 \% \mathrm{HR}_{\max }$, 255 RER $>1.15$, RPE $>18 / 20$, and blood lactate $>8 \mathrm{mmol} . \mathrm{L}^{-1}$ ), contrary to the present study. 256 Therefore, the lower ability of grade II obesity individuals to meet an $\mathrm{HR}>95 \% \mathrm{HR}_{\max }$ 257 during a treadmill test could only concern youths. According to a recent study ${ }^{28}$, 258 treadmill tests would elicit $\sim 4 \%$ lower $\mathrm{HR}_{\text {peak }}$ than actives games in children with 259 obesity. This study reinforces the idea that a high proportion of youths with obesity is 260 not achieving a maximal effort during a treadmill test.

261 The $\mathrm{VO}_{2}$ plateau used to be the primary criteria for verifying $\mathrm{VO}_{2 \max }$, but it is still 262 debated whether the occurrence of $\mathrm{VO}_{2}$ plateau is only influenced by the sampling 263 intervals, or if physiological factors could be involved ${ }^{29-31}$. In adults with obesity ${ }^{19}$, the $264 \mathrm{VO}_{2}$ plateau was reached by $46 \%$ of the participants, which is higher than the $37 \%$ 265 found in our study using the same definition of a $\mathrm{VO}_{2}$ plateau, but close to previous 266 studies in youths with ${ }^{6}$ or without ${ }^{32}$ obesity. Since the range of $\mathrm{VO}_{2}$ plateau can vary 267 from 30 to $50 \%$ between the studies $^{6,32-33}$, the present differences are probably not 268 meaningful. Furthermore, comparisons between studies might be not available since a 269 different sampling interval ${ }^{29}$ can significantly affect the results regarding the $\mathrm{VO}_{2}$ 270 plateau. 
Achievement of $\mathrm{VO}_{2 \max }$ in youths with obesity

\section{$272 \quad$ Limitations and perspectives}

273 This study has limitations. Although the methodology was based on the most recent

274 recommandations ${ }^{18,34}$, the criteria that should be used to verify $\mathrm{VO}_{2 \max }$ are still debated.

275 However, the use of supramaximal tests to verify the achievement of $\mathrm{VO}_{2 \max }$ is not

276 clinically appropriate in youths with obesity. Furthermore, the present results could

277 have been strengthened by physical activity data and the use of an RPE scale based on

278 the muscular feeling of pain. These data could have strengthened the interpretation of

279 the results regarding the mechanisms associated with the achievement of a $\mathrm{VO}_{2}$ plateau

280 or secondary threshold criteria for verifying $\mathrm{VO}_{2 \max }$. Despite these limitations, the

281 present findings are of clinical importance since performing a maximal

282 cardiorespiratory effort is the gold standard for measuring $\mathrm{VO}_{2 \max }{ }^{3}$. According to the

283 criteria used in this study and those of predecessors ${ }^{4-6}$, a high proportion of youths with

284 different grades of obesity (approximating 50\% in our study) would not $\mathrm{VO}_{2 \max }$ using a 285 treadmill walking test. Thus, clinicians should be aware that $\mathrm{VO}_{2 \max }$ may be

286 underestimated in this population when it is measured using these protocols.

\section{Conclusion}

288 The rate of achievement of an $\mathrm{HR}>95 \% \mathrm{HR}_{\max }$ and $\mathrm{RPE}>5 / 10$ ("hard") was respectively

289 independent of the grade of obesity in a sample of 72 youths. These criteria were 290 achieved by a minority of participants (15 to 64\%), suggesting that a maximal 291 cardiorespiratory effort was not achieved by the participants during the test. Youths

292 with grade II obesity displayed a lower peak in minute ventilation normalized to fat-free 293 mass and a premature feeling of difficulty than grade I during progressive walk tests, 294 which may be attributable to higher ventilatory constraints and premature feeling of leg 
Achievement of $\mathrm{VO}_{2 \max }$ in youths with obesity

295 muscular pain. Thus, our study supports the idea that a dose-response relationship exists

296 between the grade of obesity and the psychophysiological responses to an incremental

297 treadmill test in children.

\section{References}

300 1. Barry VW, Caputo JL, Kang M. The Joint Association of Fitness and Fatness on

301 Cardiovascular Disease Mortality: A Meta-Analysis. Prog Cardiovasc Dis. 2018;61(2):136-141. DOI:10.1016/j.pcad.2018.07.004

2. Imboden MT, Kaminsky LA, Peterman JE, et al. Cardiorespiratory Fitness Normalized to Fat-Free Mass and Mortality Risk. Med Sci Sports Exerc. 2020;52(7):1532-1537. DOI:10.1249/MSS.0000000000002289

3. Poole DC, Richardson RS. Determinants of oxygen uptake. Implications for exercise testing. Sports Med. 1997;24(5):308-320. DOI:10.2165/00007256199724050-00003

4. Gutin B, Barbeau P, Owens S, et al. Effects of exercise intensity on cardiovascular fitness, total body composition, and visceral adiposity of obese adolescents. Am J Clin Nutr. 2002;75(5):818-826. DOI:10.1093/ajen/75.5.818

5. Marinov B, Kostianev S, Turnovska T. Ventilatory efficiency and rate of perceived exertion in obese and non-obese children performing standardized exercise. Clin Physiol Funct Imaging. 2002;22(4):254-260. DOI:10.1046/j.1475097x.2002.00427.x

6. Breithaupt PG, Colley RC, Adamo KB. Using the oxygen uptake efficiency slope as an indicator of cardiorespiratory fitness in the obese pediatric population. Pediatr Exerc Sci. 2012;24(3):357-368. DOI:10.1123/pes.24.3.357 
7. Peyrot N, Thivel D, Isacco L, Morin JB, Belli A, Duche P. Why does walking economy improve after weight loss in obese adolescents?. Med Sci Sports Exerc. 2012;44(4):659-665. DOI:10.1249/MSS.0b013e318236edd8

8. Thivel D, Masurier $\mathrm{J}$, Baquet $\mathrm{G}$, et al. High-intensity interval training in overweight and obese children and adolescents: systematic review and metaanalysis. J Sports Med Phys Fitness. 2019;59(2):310-324. DOI:10.23736/S00224707.18.08075-1

9. Cole TJ, Lobstein T. Extended international (IOTF) body mass index cut-offs for thinness, overweight and obesity. Pediatr Obes. 2012;7(4):284-294. DOI:10.1111/j.2047-6310.2012.00064.X

10. de Onis M, Onyango AW, Borghi E, Siyam A, Nishida C, Siekmann J. Development of a WHO growth reference for school-aged children and adolescents. Bull World Health Organ. 2007;85(9):660-667. DOI:10.2471/blt.07.043497

11. Mirwald RL, Baxter-Jones AD, Bailey DA, Beunen GP. An assessment of maturity from anthropometric measurements. Med Sci Sports Exerc. 2002;34(4):689-694. DOI:10.1097/00005768-200204000-00020

12. Tanaka H, Monahan KD, Seals DR. Age-predicted maximal heart rate revisited. J Am Coll Cardiol. 2001;37(1):153-156. DOI:10.1016/s0735-1097(00)01054-8 determination of maximal oxygen uptake: a brief critique and recommendations for future research. Sports Med. 2007;37(12):1019-1028. DOI:10.2165/00007256-200737120-00002

14. Midgley AW, Bentley DJ, Luttikholt H, McNaughton LR, Millet GP. Challenging a dogma of exercise physiology: does an incremental exercise test 
for valid VO 2 max determination really need to last between 8 and 12 minutes?. Sports Med. 2008;38(6):441-447. DOI:10.2165/00007256-200838060-00001

15. Hermand

E, Cassirame J, Ennequin

G, Hue

O. Validation of a Photoplethysmographic Heart Rate Monitor: Polar OH1. Int J Sports Med. 2019;40(7):462-467. DOI:10.1055/a-0875-4033

16. Blanchard J, Blais S, Chetaille P, et al. Determination of peak cardiorespiratory fitness parameters in children: which averaging method should we use?. J Sports Sci. 2019;37(11):1265-1269. DOI:10.1080/02640414.2018.1554976

17. Williams JG, Eston R, Furlong B. CERT: a perceived exertion scale for young children. Percept Mot Skills. 1994;79(3 Pt 2):1451-1458. DOI:10.2466/pms.1994.79.3f.1451

18. Niemeyer M, Bergmann TGJ, Beneke R. Oxygen uptake plateau: calculation artifact or physiological reality?. Eur J Appl Physiol. 2020;120(1):231-242. DOI:10.1007/s00421-019-04267-7

19. Wood RE, Hills AP, Hunter GR, King NA, Byrne NM. $\mathrm{Vo}_{2 \max }$ in overweight and obese adults: do they meet the threshold criteria?. Med Sci Sports Exerc. 2010;42(3):470-477. DOI:10.1249/MSS.0b013e3181b666ad

20. Poole DC, Jones AM. Measurement of the maximum oxygen uptake $\dot{V}_{2}$ max $\dot{V}_{o_{2 p e a k}}$ is no longer acceptable. J Appl Physiol (1985). 2017;122(4):997-1002. DOI:10.1152/japplphysiol.01063.2016

21. Mendelson M, Michallet AS, Tonini J, Favre-Juvin A, Guinot M, Wuyam B, Flore P. Low Cardiorespiratory Fitness is Partially Linked to Ventilatory Factors in Obese Adolescents. Pediatr Exerc Sci. 2016 Feb;28(1):87-97. doi: 10.1123/pes.2013-0151. Epub 2015 Aug 25. PMID: 26305371. 
22. Thivel D, Isacco L, O'Malley G, Duché P. Pediatric Obesity and Perceived Exertion: Difference Between Weight-Bearing and Non-Weight-Bearing Exercises Performed at Different Intensities. J Sports Sci. 2016;34(5):389-394. DOI:10.1080/02640414.2015.1061200

23. Thivel D, O'Malley G, Blourdier D, Tremeaux M, Zanchet C, Pereira B, Ratel S. Reproducibility of the intermittent Spartacus Run Test in obese adolescents. J Sports Med Phys Fitness. 2017 Sep;57(9):1083-1088. doi: 10.23736/S00224707.16.06534-8. Epub 2016 Jul 7. PMID: 27387496.

24. O'Malley G, Hussey J, Roche E. A pilot study to profile the lower limb musculoskeletal health in children with obesity. Pediatr Phys Ther. 2012;24(3):292-298. DOI:10.1097/PEP.0b013e31825c14f8

25. Quinart S, Groslambert A, Ecarnot F, Simon-Rigaud ML, Nicolet-Guénat M, Nègre V, Mougin F. Validation of a new scale to measure perceived exertion in obese adolescents: the Childhood Obesity Perceived Exertion (Cope-10) Scale. J Sports Med Phys Fitness. 2016 Sep;56(9):1013-20. Epub 2015 Jul 1. PMID: 26129916.

26. Robertson RJ, Goss FL, Boer NF, Peoples JA, Foreman AJ, Dabayebeh IM, Millich NB, Balasekaran G, Riechman SE, Gallagher JD, Thompkins T. Children's OMNI scale of perceived exertion: mixed gender and race validation. Hadjiyannakis S, Colley RC, Adamo KB. Do obese children perceive submaximal and maximal exertion differently? Clin Med Insights Pediatr. 2013 
Sep 8;7:35-40. doi: 10.4137/CMPed.S12524. PMID: 24052695; PMCID: PMC3775604.

28. Berntsen S, Edvardsen E, Gerbi S, Kolsgaard ML, Anderssen SA. Do Obese Children Achieve Maximal Heart Rate during Treadmill Running? Sports (Basel). 2019 Jan 19;7(1):26. doi: 10.3390/sports7010026. PMID: 30669424; PMCID: PMC6359420.

29. Astorino TA. Alterations in VOmax and the VO plateau with manipulation of sampling interval. Clin Physiol Funct Imaging. 2009 Jan;29(1):60-7. doi: 10.1111/j.1475-097X.2008.00835.x. PMID: 19125732.

401

402

403

404

405

406

407

408

409

410

411

412

30. Beltrami FG, Wong del P, Noakes TD. High prevalence of false-positive plateau phenomena during VO2max testing in adolescents. J Sci Med Sport. 2014 Sep;17(5):526-30. doi: 10.1016/j.jsams.2013.07.012. Epub 2013 Aug 8. PMID: 23992661.

31. Niemeyer M, Bergmann TGJ, Beneke R. Oxygen uptake plateau: calculation artifact or physiological reality? Eur J Appl Physiol. 2020 Jan;120(1):231-242. doi: 10.1007/s00421-019-04267-7. Epub 2019 Nov 20. PMID: 31748882.

32. Sansum KM, Weston ME, Bond B, Cockcroft EJ, O'Connor A, Tomlinson OW, Williams CA, Barker AR. Validity of the Supramaximal Test to Verify Maximal Oxygen Uptake in Children and Adolescents. Pediatr Exerc Sci. 2019 May 1;31(2):213-222. doi: 10.1123/pes.2018-0129. Epub 2019 Mar 18. PMID: 30885084.z 
Achievement of $\mathrm{VO}_{2 \max }$ in youths with obesity

413 33. Rowland TW, Cunningham LN. Oxygen uptake plateau during maximal 414 treadmill exercise in children. Chest. 1992 Feb;101(2):485-9. doi: 10.1378/chest.101.2.485. PMID: 1735277.

416 34. Marinus N, Bervoets L, Massa G, et al. Altered gas-exchange at peak exercise in obese adolescents: implications for verification of effort during cardiopulmonary exercise testing. J Sports Med Phys Fitness. 2017;57(12):1687-1694. DOI:10.23736/S0022-4707.16.06607-X 
Achievement of $\mathrm{VO}_{2 \max }$ in youths with obesity

421 Table 1 : Participants' characteristics, cardiorespiratory and perceptual responses by 422 grade of obesity subgroups.

\begin{tabular}{|c|c|c|}
\hline & $\begin{array}{l}\text { IOTF Grade I obesity } \\
\qquad(\mathrm{n}=15)\end{array}$ & $\begin{array}{l}\text { IOTF Grade II obesity } \\
\qquad(\mathrm{n}=57)\end{array}$ \\
\hline $\begin{array}{l}\text { Age } \\
\text { (years) }\end{array}$ & $11.9 \pm 1.8$ & $11.6 \pm 1.8$ \\
\hline $\begin{array}{l}\text { Age of PHV } \\
\text { (years) }\end{array}$ & $12.8 \pm 1.2$ & $12.0 \pm 0.9^{*}$ \\
\hline $\begin{array}{l}\text { Maturity } \\
\text { (years from PHV) }\end{array}$ & $-0.9 \pm 1.1$ & $-0.4 \pm 1.8$ \\
\hline $\begin{array}{l}\text { Height } \\
(\mathrm{cm})\end{array}$ & $152 \pm 10$ & $153 \pm 11$ \\
\hline $\begin{array}{l}\text { Weight } \\
(\mathrm{kg})\end{array}$ & $56.0 \pm 11.7$ & $70 \pm 18.8^{* * *}$ \\
\hline $\begin{array}{l}\text { Body Mass Index } \\
\left(\mathrm{kg} \cdot \mathrm{m}^{-2}\right)\end{array}$ & $23.9 \pm 2.2$ & $29.8 \pm 4.6^{* * *}$ \\
\hline $\begin{array}{l}\text { Fat mass } \\
\text { (\% body mass) }\end{array}$ & $28.7 \pm 4.4$ & $39.1 \pm 7.1^{* * *}$ \\
\hline $\begin{array}{l}\text { IOTF BMI z-score } \\
\text { (SD) }\end{array}$ & $1.8 \pm 0.2$ & $2.7 \pm 0.5^{* * * *}$ \\
\hline $\begin{array}{l}\mathrm{VE}_{\text {peak }} \\
\mathrm{mL} \cdot \mathrm{kgFFM}^{-1} \cdot \mathrm{min}^{-1}\end{array}$ & $1.94 \pm 0.33$ & $1.71 \pm 0.31^{*}$ \\
\hline $\begin{array}{l}\mathrm{VE}_{\text {peak }} \\
\mathrm{mL} \cdot \mathrm{kgBM}^{-1} \cdot \mathrm{min}^{-1}\end{array}$ & $1.39 \pm 0.26$ & $1.04 \pm 0.24^{* * *}$ \\
\hline $\begin{array}{l}\mathrm{VO}_{2 \text { peakBM }} \\
\left(\mathrm{mL} \cdot \mathrm{kgBM}^{-1} \cdot \mathrm{min}^{-1}\right)\end{array}$ & $34.5 \pm 7.1$ & $27.2 \pm 5.1^{* *}$ \\
\hline 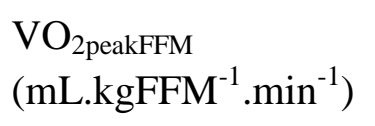 & $48.2 \pm 8.7$ & $44.8 \pm 7 . .4$ \\
\hline $\begin{array}{l}\mathrm{HR}_{\text {peak }} \\
\text { (bpm) }\end{array}$ & $192 \pm 14$ & $185 \pm 13$ \\
\hline $\begin{array}{l}\mathrm{HR}_{\text {peak }} \\
\left(\% \text { predicted } \mathrm{HR}_{\max }\right)\end{array}$ & $96.2 \pm 7.1$ & $92.5 \pm 6.7$ \\
\hline $\begin{array}{l}\mathrm{HR}_{\mathrm{RPEhard}} \\
\left(\% \mathrm{HR}_{\text {peak }}\right)\end{array}$ & $93.3 \pm 6.2$ & $94.1 \pm 4.7$ \\
\hline $\begin{array}{l}\mathrm{RER}_{\text {peak }} \\
\text { (Arbitrary unit) }\end{array}$ & $1.10 \pm 0.17$ & $1.08 \pm 0.14$ \\
\hline
\end{tabular}


Achievement of $\mathrm{VO}_{2 \max }$ in youths with obesity

424 Table 2 : Number and rate of participants having reached the criteria for verying $\mathrm{VO}_{2 \max }$ 425 and achieved $\mathrm{VO}_{2 \max }$ according to these criteria, by grade of obesity and results of 426 logistic regressions.

\begin{tabular}{|c|c|c|c|c|c|c|c|c|c|c|}
\hline & \multicolumn{2}{|c|}{$\mathrm{VO}_{2 \max }$} & \multicolumn{2}{|c|}{$\mathrm{VO}_{2}$ plateau } & \multicolumn{2}{|c|}{$\mathrm{HR}>95 \%$} & \multicolumn{2}{|c|}{ RER $>1.0$} & \multicolumn{2}{|c|}{$\mathrm{RPE}>5 / 10$} \\
\hline & Yes & No & Yes & No & Yes & No & Yes & No & Yes & No \\
\hline Grade & $\mathrm{n}=7$ & $\mathrm{n}=8$ & $\mathrm{n}=4$ & $\mathrm{n}=11$ & $\mathrm{n}=9$ & $\mathrm{n}=6$ & $\mathrm{n}=8$ & $\mathrm{n}=7$ & $\mathrm{n}=12$ & $\mathrm{n}=3$ \\
\hline I & $47 \%$ & $53 \%$ & $27 \%$ & $73 \%$ & $60 \%$ & $40 \%$ & $53 \%$ & $47 \%$ & $80 \%$ & $20 \%$ \\
\hline Grade & $n=33$ & $n=24$ & $n=23$ & $n=34$ & $n=19$ & $\mathrm{n}=38$ & $n=39$ & $n=18$ & $\mathrm{n}=54$ & $n=3$ \\
\hline II & $58 \%$ & $42 \%$ & $40 \%$ & $60 \%$ & $33 \%$ & $67 \%$ & $68 \%$ & $32 \%$ & $95 \%$ & $5 \%$ \\
\hline $\begin{array}{c}\mathrm{X}^{2} \\
\text { tests }\end{array}$ & \multicolumn{2}{|c|}{$P=0.44$} & \multicolumn{2}{|c|}{$P=0.33$} & \multicolumn{2}{|c|}{$P=0.06$} & \multicolumn{2}{|c|}{$P=0.27$} & \multicolumn{2}{|c|}{$P=0.07$} \\
\hline OR & \multicolumn{2}{|c|}{ NS } & \multicolumn{2}{|c|}{ NS } & \multicolumn{2}{|c|}{0.33} & \multicolumn{2}{|c|}{ NS } & \multicolumn{2}{|c|}{4.5} \\
\hline
\end{tabular}

